\title{
Microfluidic synthesis of retinoic acid-loaded nanoparticles for neural differentiation of trabecular meshwork mesenchymal stem cells
}

\author{
Mohammadi $\mathrm{P}^{1}$, Rahmani $\mathrm{A}^{2}$, Habibizadeh $\mathrm{M}^{3}$, Nadri $\mathrm{S}^{2,4,5}$ \\ Medical Nanotechnology Department Zanjan, Iran. nadrisamad@gmail.com
}

\begin{abstract}
OBJECTIVES: This study aimed to fabricate the PCL-nanoparticles (NPS) loaded retinoic acid (RA) using the microfluidic system for successful cellular uptake and induction of neuronal differentiation of trabecular meshwork mesenchymal stem cells (TMMSCs).

METHODS: A microfluidic system used to synthesize RA-loaded NPS, DLS, FTIR, TEM, and UVspectroscopy was recruited to characterize and study the release of RA. Also, the toxicity, cellular uptake, and neuronal differential of TMMSCs have been assessed.

RESULTS: According to the obtained results, the spherical NPs (117.6 $\pm 0.35 \mathrm{~nm},-19.4 \pm 5.3)$ and RA-loaded NPs $(121.6 \pm 0.75 \mathrm{~nm},-23.6 \pm 1.3)$ were synthesized successfully by microfluidic system. $7.8 \pm 2.04 \%$ of RA was loaded in NPs, and $25 \%$ was released in the first four hours. Thus, the NPs have been successfully internalized into the stem cells, leading to a significant increase in neural genes and protein ( $\beta$ Tubulin III and Map-2) expression.

CONCLUSION: Our study's harvested results have represented valid data for practical use of microfluidic systems in the term of NPs loaded RA synthesis and its successful function to cellular internalization and euronal differentiation of TMMSCs (Tab. 2, Fig. 10, Ref. 46). Text in PDF www.elis.sk KEY WORDS: microfluidic, RA-loaded nanoparticles, neural differentiation, MSCs.
\end{abstract}

\section{Introduction}

Nanoparticles (NPs) have great potential to enhance the therapeutic effects of pharmaceutical agents through enhancing cellular entrance, bioavailability, biodistribution, and raising the endosomal and lysosomal escape (1-5). To date, various techniques such as precipitation and hydrothermal have been used for NPs synthesis (6). However, due to the low reproducibility (in size and polydispersity index (PDI)), the nanoparticles could be attributed to uncontrollable processes of mixing and separation in the conventional methods. The microfluidic system is a newly emerging field

${ }^{1}$ Department of Medical Biotechnology, School of Medicine, Zanjan University of Medical Sciences, Zanjan, Iran, ${ }^{2}$ Department of Medical Nanotechnology, School of Medicine, Zanjan University of Medical Sciences, Zanjan, Iran, ${ }^{3}$ Department of Pharmacy Biomaterial, School of Pharmacy, Zanjan University of Medical Sciences, Zanjan, Iran, ${ }^{4}$ Zanjan Metabolic Diseases Research Center, Zanjan University of Medical Sciences, Zanjan, Iran, and ${ }^{5}$ Zanjan Pharmaceutical Nanotechnology Research Center, Zanjan University of Medical Sciences, Zanjan, Iran

Address for correspondence: S. Nadri, PhD, Medical Nanotechnology Department, P.O.Box: 14115-111, Zanjan, Iran.

Acknowledgements: This study was supported by Elite Researcher Grant Committee under award number [Grant No: 977035] from the National Institutes for Medical Research Development (NIMAD), under Biotechnology Research ethics committees of National Institute for Medical Research and Development (Ethical NO: IR.NIMAD.REC.1397.414). that overcomes the limitation of traditional methods in the view of NPs synthesis (7-10).

Microfluidic systems are a new approach to fabricate the NPs more appropriately. In the microfluidic system, the phases of nucleation and growth of nanoparticle formation have been isolated; thus, it controls exceedingly size, PDI, morphology, and thus increased reproducibility $(7,11-13)$. When compared to the bulk methods, the microfluidic technology also represented more advantages such as homogenous reaction environments (14), lower consumption of expensive agents (15), and high throughput analysis, fast and constant (16). These methods were used to produce the drug-loaded polymeric nanoparticles (PNPs) (17).

The PNPs may be the best choice to fabricate a system for a growth factor (GF) delivery in regenerative medicine because of the proper biocompatibility, low cytotoxicity, and enzymatic degradation protection of GF (18-20). GF biodistribution and spatial distribution strongly affect critical biological processes such as cell differentiation, proliferation, and migration. So, the GF delivery system can play a vital role in regenerative medicine efficiency (21). Retinoic acid (RA) is a metabolite of vitamin A that mediates the functions of vitamin A required for induction of nervous system regeneration, the treatment of neurodegenerative diseases, growth and development of tissue and stem cells (22-24). Mesenchymal stem cells (MSCs) are the best candidate for cell therapy of many neurological diseases $(25,26)$. Numerous factors 
Tab. 1. The DLS obtained data for NPs diameter, PDI and Z-potential.

\begin{tabular}{lccc}
\hline & Diameter $(\mathrm{nm})$ & PDI & Z-potential \\
\hline PCL-NPs & $117.6 \pm 0.35$ & $0.174 \pm 0.01$ & $-19.4 \pm 5.3$ \\
RA-loaded PCL NPs & $121.6 \pm 0.75$ & $0.201 \pm 0.02$ & $-23.6 \pm 1.3$ \\
\hline
\end{tabular}

such as RA have reported the differentiation of MSCs into neural cells (27). However, several limitations have been attributed to the raw RA administration, including low solubility and rapid cell metabolization (28). Hence, finding new applicable approaches to remove the obstacles toward the in vivo application of $R A$ seems necessary. Due to some challenges encountering RA delivery, such as low-transporting volume and low-throughput of the entrapping methods, we assumed that RA entrapping in NPs using a microfluidic device might overcome the barriers.

However, in the present study, we aimed to fabricate PCLNPs loaded RA using microfluidic systems as a high-throughput method to deliver RA more effectively for neural differentiation of trabecular meshwork mesenchymal stem cells (TMMSCs).

\section{Material and methods}

\section{Microfluidic device fabrication}

The soft lithographic technique is used for microfluidic device fabrication. Briefly, the silica-wafer was spun $(3,000 \mathrm{rpm}$ for 5 min) with negative photoresist SU8 (3050; Merk) in a cleanroom environment, followed by a pre-baking on a hotplate $\left(65^{\circ} \mathrm{C}, 60\right.$ $\mathrm{min})$. Then, UV irradiation ( $330-440 \mathrm{~nm}$ ) was exposed on the wafer coated with a patterned mask. After the post-baking $\left(95^{\circ} \mathrm{C}, 10\right.$ $\mathrm{min}$ ), the wafer was submerged in a developer, followed by drying with neutral gas. For replica molding, the PDMS (Sylgard, Dow Corning) and curing agent were put together (10:1 w/w ratio) and cast on channel masters to form the PDMS channels and then baked at $70{ }^{\circ} \mathrm{C}$ for 3 hours. Finally, the cured PDMS channels are peeled with SU-8 master. The bonding strength was provided by pre-treating the contact surfaces with oxygen plasma for $60 \mathrm{~s}$ in the plasma cleaner (Merk co). The inlets and outlets were made using a $1.5 \mathrm{~mm}$ biopsy punch.

\section{Preparation of RA-loaded PCL nanoparticles}

Polycaprolactone (PCL, Merk, $\mathrm{Mn}=80,000$ ) was dissolved in acetone (AMT 100013, Ameretatshimi co, Iran) at $45^{\circ} \mathrm{C}$ for $1 \mathrm{~h}$, by the concentration of $1 \% \mathrm{w} / \mathrm{v}$. The obtained solution was diluted to the final concentration of $0.2 \% \mathrm{v} / \mathrm{v}$ for nanoparticle fabrication. To synthesize RA-loaded nanoparticles (RA-PCL NPs), the RA was added to the $0.2 \%$ solution by the concentration of $10 \mu \mathrm{M}$ and stirred for 10 minutes. Tween 80 was dissolved by the concentration of $1 \% \mathrm{w} / \mathrm{v}$ in double-distilled water $(\mathrm{ddH} 2 \mathrm{O})$ and used as the surfactant in the aqueous phase. PCL, PCL-RA, and water/tween 80 solutions were loaded in $5 \mathrm{ml}$ syringes separately and conducted in the micropump with a flow rate of $2 \mathrm{ml} / \mathrm{h}$ for the polymeric phase and $10 \mathrm{ml} / \mathrm{h}$ for the aqueous phase. The fabricated nanoparticles were harvested in a microtube for subsequent analysis.

\section{Characterization of NPs}

The average size and polydispersity index (PDI) of NPs are determined by a zeta-sizer (Malvern Instruments, Malvern, UK). The NPs samples were diluted (in distilled water) and analyzed at $25{ }^{\circ} \mathrm{C}$. Furthermore, to evaluate NPs morphology, the dilute aqueous solution of NPs was dropped on copper grid mesh (EMSUSA) and observed by transmission electron microscopy (TEM, model Zeiss-EM10C Company) at an accelerating voltage of 100 $\mathrm{kV}$. The chemical composition and bond structures of any component evaluated by attenuated total reflectance-Fourier transforms infrared spectroscopy (ATR-FTIR) at a resolution of $4 \mathrm{~cm}^{-1}$ in the range from 3000 to $400 \mathrm{~cm}^{-1}$.

\section{Drug loading}

To take out any non-encapsulated RA from the RA-PCL NPs solution, ultrafiltration (MWCO $=30 \mathrm{kDa}$, Amicon ${ }^{\mathrm{TM}}$ Ultra-4) was used. The collected RA-PCL NPs were centrifuged (30 min at $18,000 \mathrm{rpm}$ ), dried (in an oven at $60^{\circ} \mathrm{C}$ for $12 \mathrm{~h}$ ), weighted, and
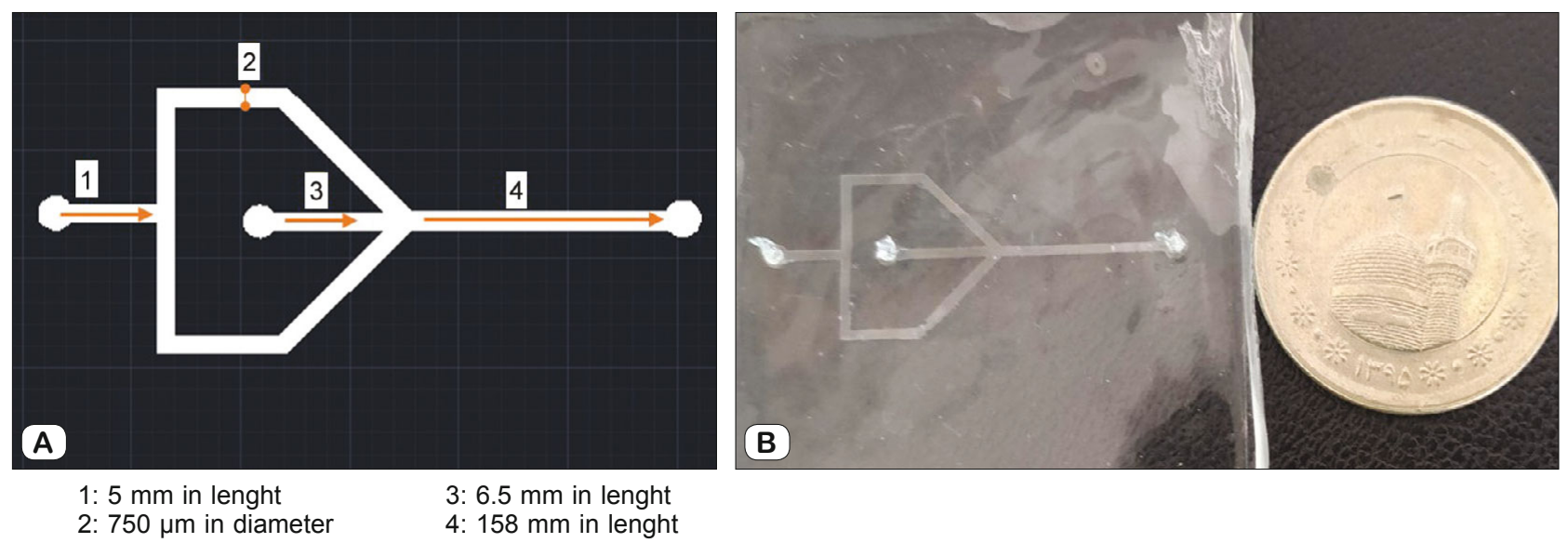

Fig. 1. Soft lithography fabricated microchip device. A: Design of chip and length of channels. B: The chip is transparent and contains two inlets (for water and PCL) and an outlet to extrude nanoparticles. 


\section{4-891}

solved in DMSO/chloroform solvent. Subsequently, the loaded RA was determined by measuring the drug concentration in the RA-NPs DMSO/chloroform solution by UV-Vis spectrophotometry at a wavelength of $345 \mathrm{~nm}$. The RA loading was calculated using the equation:

Drug Loading $(\%)=\frac{\text { Amount of the drug in NPs }}{\text { Total weight of NPs }} \times 100$

\section{$R A$ release studies}

The concentrated RA-PCL NPs solution (by an ultra-centrifugal filter) was located into a dialysis bag (cut off $12 \mathrm{kDa}$ ) and poured into $5 \mathrm{~mL}$ of PBS under stirring at $37^{\circ} \mathrm{C}$. At predetermined time intervals, $0.5 \mathrm{~mL}$ of PBS was taken out and substituted with fresh PBS. Then, the drug concentration was determined by UV-VIS spectroscopy.

\section{PCL- NPs biocompatibility}

Cell viability assay: Trabecular Meshwork Mesenchymal Stem Cell (TMMSCs) were isolated, characterized, and cultured according to a protocol modified by Nadri et al (29). PCL- NPs were diluted to $100 \mathrm{ml}$ in rich DMEM $(30 \mu \mathrm{g} / \mathrm{ml})$ and added into the cell culture media. MTT assay was performed at 1,3 , and 5 days after additions of NPs to the cell culture medium. Briefly, the MTT solution was prepared using $5 \mathrm{mg} / \mathrm{ml}$ in DMEM and poured into samples followed by $3-4 \mathrm{~h}$ incubation. To solubilize the formazan crystals, dimethylsulfoxide (DMSO) was added to the solution absorbance read at $570 \mathrm{~nm}$ using a Microplate Reader (ELX800; BioTeK, Winooski, VT).

Hemolysis assay: To assess the hemocompatibility of NPs, the hemolysis assay was performed. The fresh human blood samples were obtained from healthy volunteers (After informed consent was obtained from the individual and under approval of Biotechnology Research ethics committees of the National Institute for Medical Research and Development (Ethical NO: IR.NIMAD. REC.1397.414)). Initially, $5 \mathrm{ml}$ blood was poured into tubes containing EDTA and then centrifuged at $4000 \mathrm{rpm}$ for $15 \mathrm{~min}$ to precipitate the human red blood cells. The supernatant plasma surface layer was removed and deposited red blood cells (RBC) pellets were separated and washed with the sterile PBS solution. $0.5 \mathrm{ml}$ diluted blood added to $0.5 \mathrm{ml}$ of PCL-NPs (concentrations between $200 \mu \mathrm{g} / \mathrm{ml}$ and $12.5 \mu \mathrm{g} / \mathrm{ml}$ ), $0.5 \mathrm{~mL}$ of deionized water as a positive control, and $0.5 \mathrm{~mL}$ of PBS as a negative control. The tubes were incubated at $37^{\circ} \mathrm{C}$ for $3 \mathrm{~h}$ with gentle inversion of the sample tubes every $30 \mathrm{~min}$, centrifuged at 13,000 rpm for 15 min to isolate non-lysed human red blood cells, and absorbance of samples has been measured spectrophotometrically at $540 \mathrm{~nm}$. The percentage of hemolysis was calculated for each sample with the equation: (Abs, i.e., absorbance)

Hemolysis $(\%)=\frac{\text { Abas Sample }- \text { Abs Negative control }}{\text { Abs Positive Control }- \text { Abs Negative Control }}$

\section{Neural differentiation}

NPs cell Uptake: The cellular uptake of PCL-NPs in TMMSCs cells was analyzed by the fluorescence method. In brief, the cells were cultivated on a chambered cover glass $\left(10^{3}\right.$ cells $)$ and in- cubated with curcumin loaded nanoparticle $(50 \mu \mathrm{g} / \mathrm{mL})$ medium for 2 hours and washed with phosphate-buffered solution (PBS), stained with 4',6-diamidino-2-phenylindole (Sigma) DAPI $(10 \mu \mathrm{g} / \mathrm{mL})$. The cells were observed by fluorescent microscopy (OLYMPUS, BX51) with differential contrast channels (blue (DAPI) with excitation at $340 \mathrm{~nm}$ and a yellow (curcumin) with excitation at $488 \mathrm{~nm})$.

Real Time-qPCR for neural marker genes: To investigate the neuronal differentiation of TMMSCs, the Real Time-qPCR (RTqPCR) was performed. The total RNA extracted by RNAX PLUS (Sinaclon, Iran). Quantification and purity of RNA were determined using a spectrophotometer (nanodrop 2000, Wilmington, USA), and cDNA was synthesized using BioFact RT Series cDNA synthesis Kit (BioFact, Korea) according to the manufacturer's procedure. Real-time PCR was performed with an Applied Biosystems TM Real-Time PCR System (Life Technologies Corporation, USA) for all genes (Beta Tubulin, Map-2, Nestin) for 40 cycles (Tab. 1).

Immunostaining (ICC): The samples were fixed (4\% paraformaldehyde), treated with $0.5 \%$ Triton X-100, and incubated with primary antibodies ( $\beta$ Tubulin III, MAP-2) at $4{ }^{\circ} \mathrm{C}$. In the next step, the samples were incubated with the FITC-conjugated $\mathrm{IgG}$ (Sigma) and $0.1 \mu \mathrm{g} / \mathrm{ml}$ DAPI. Finally, FITC-conjugated-cells were observed using an inverted fluorescence microscope.

\section{Statistical analyses}

One-way analysis of variance was used to analyze data using GraphPad Prism 6 software (Graphpad Software Inc., La Jolla,
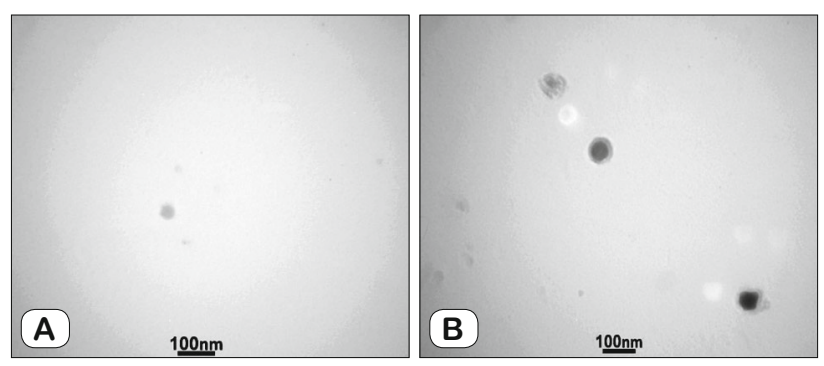

Fig. 2. TEM images of PCL-NPs (A, B) and RA-loaded PCL-NPs (C, D).

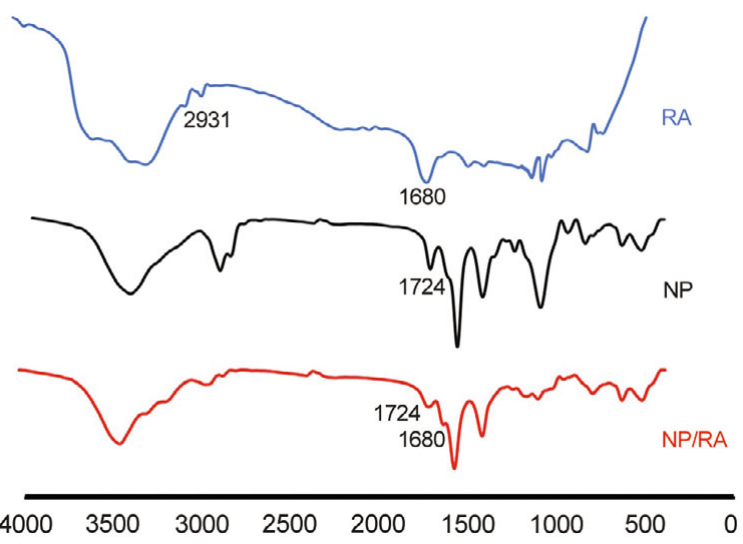

Fig. 3.The FTIR spectrums of RA, PCL-NPs, and RA-loaded PCL-NPs. The specific peaks in each spectrum were indicated. 
CA). Data were represented as mean \pm standard deviation (SD). $\mathrm{p}<0.05$ was considered a statistically significant level.

\section{Results}

Fabrication of a microfluidic device

The designed chip and the fabricated microchip are shown in Figure 1. According to the designed parameter, the diameter of the channels was $750 \mathrm{um}$, and the mixing channel length was $15 \mathrm{~mm}$.

\section{Characterization of drug-loaded nanoparticles}

The chemical composition, morphological properties, and diameter of NP were analyzed by FTIR, TEM, and DLS, respectively. The TEM images showed that nanoparticles have a uniform size distribution for both PCL and RA- loaded NPs (Fig. 2). The RA, PCLNPs, and PCL-RA-NPs FTIR spectrums are shown in Figure 3, and the prominent peaks were represented for each. The peak at $1724 \mathrm{~cm}^{-1}$ as the distinct central band for PCL represented the carbonyl group stretching absorption (30). The indicated band at $1680 \mathrm{~cm}^{-1}$ and $2931 \mathrm{~cm}^{-1}$ in the RA spectra attributed to the carbonyl group and its aliphatic alkanes of RA, respectively (31). All the indicated spectra in RA and PCL-NP were presented in the RA- loaded PCL NPs, representing the RA loading in the NPs. According to the data (Tab. 2 and Fig. 4), the mean diameter and Zpotential for PCL-NPs and RA- loaded PCL NPs were 117.6 \pm 0.35 $\mathrm{nm},-19.4 \pm 5.3$, and $121.6 \pm 0.75 \mathrm{~nm},-23.6 \pm 1.3$, respectively.

\section{$R A$ loading and release}

The results showed that the loading rate of RA in NPs was $7.8 \pm 2.04 \%$ ( $\approx 0.2 \mathrm{uM} \mathrm{RA}$ ), and the In vitro release assay demonstrated that the RA-loaded PCL NPs released just $25 \%$ of the total loaded RA in the first $4 \mathrm{~h}$ (Fig. 5).
(A)

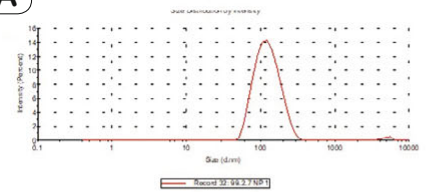

C

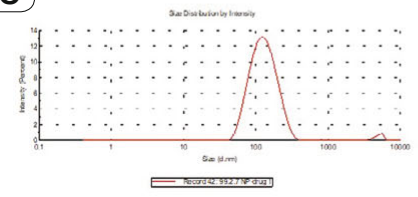

(B)

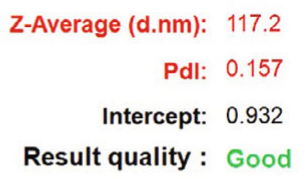

Result quality : Good

(D)

$$
\begin{aligned}
\text { Z-Average (d.nm): } & 120.7 \\
\text { Pdl: } & 0.201 \\
\text { Intercept: } & 0.938 \\
\text { Result quality : } & \text { Good }
\end{aligned}
$$

Zeta Potential (mV): -22.5

Zeta Deviation $(\mathrm{mV}): 3.27$

Conductivity $(\mathrm{ms} / \mathrm{cm}): 0.0280$

Result quality : Good

Fig. 4. The DLS analysis. Diameter, PDI, and Z-potential of PCL-NPs (A, B) and RA-loaded PCL-NPs (C, D).

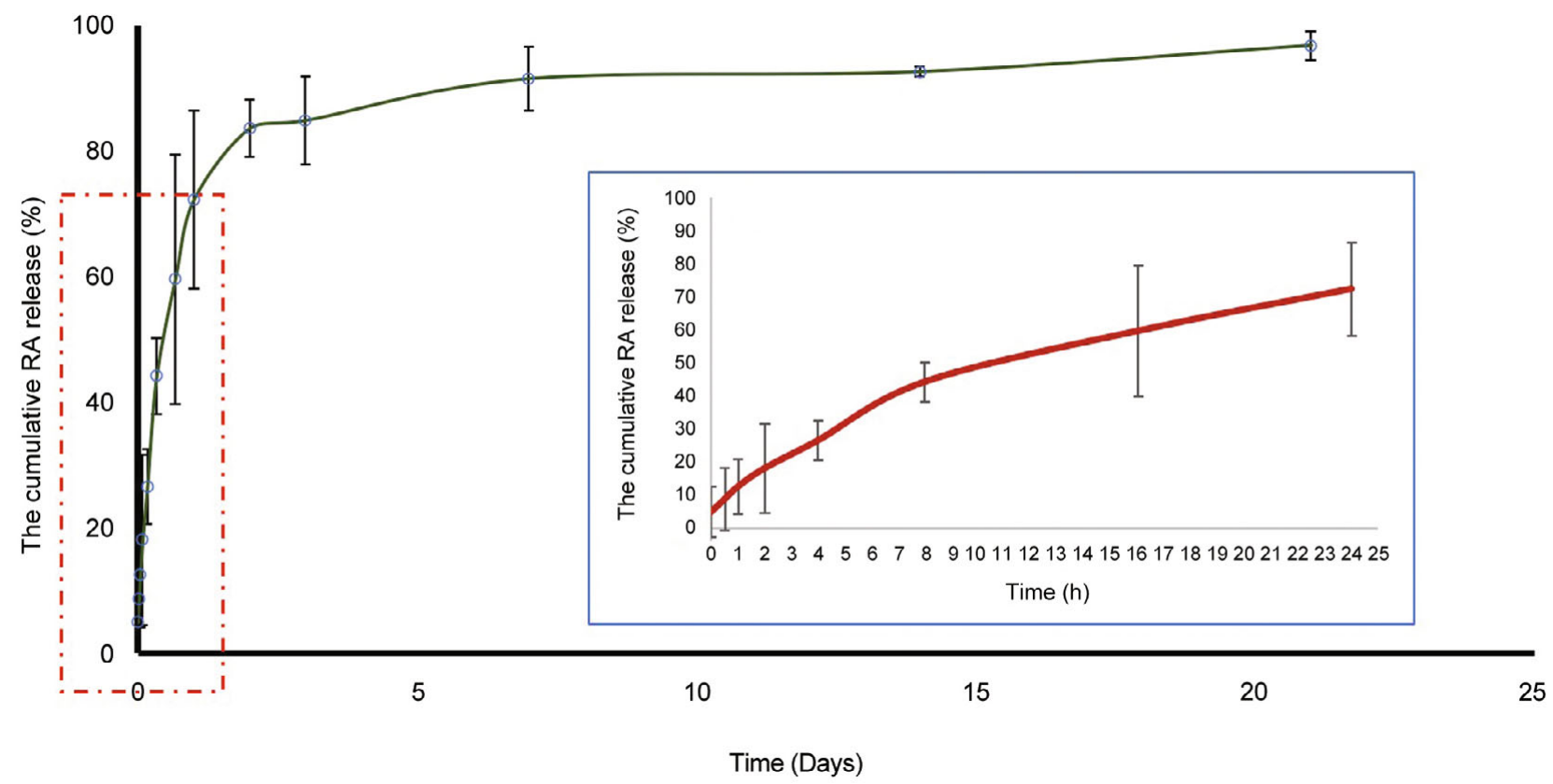

Fig. 5. In vitro RA release profile. A: 21 days of RA-loaded PCL-NPs B: The enlarged Fig. of the release profile in the first 24 hours. 
884-891

Tab. 2. Neural gene primers used for RT-qPCR.

\begin{tabular}{llc}
\hline Genes & Primer sequences & Size (bp) \\
\hline \multirow{2}{*}{ BIII- Tubulin } & $\begin{array}{l}\text { F: TGGAGTGAGAGAGGCAGGTG } \\
\text { R: GTGTCGGCAGCAAGATGG }\end{array}$ & 76 \\
\hline \multirow{2}{*}{ Nestin } & $\begin{array}{l}\text { F: GAAGGTGAAGGGCAAATCTG } \\
\text { R: CCTCTTCTTCCCATATTTCCTG }\end{array}$ & 96 \\
\hline MAP-2 & $\begin{array}{l}\text { F: AGTTCCAGCAGCGTGATG } \\
\text { R: CATTCTCTCTTCAGCCTTCTC }\end{array}$ & 97 \\
\hline GAPDH & $\begin{array}{l}\text { F: GTGAACCATGAGAAGTATGACAAC } \\
\text { R: CATGAGTCCTTCCACGATACC }\end{array}$ & \multirow{2}{*}{123} \\
\hline
\end{tabular}

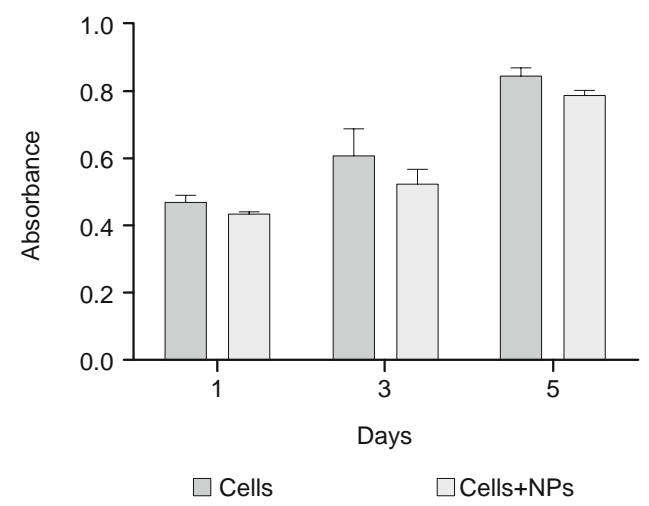

Fig. 6. The cell viability of TMMSCs. Data represented as mean \pm SD. SD: Standard Deviation.

\section{PCL- NPs biocompatibility}

To determine the cytotoxicity of these nanoparticles, TMMSCs were exposed to PCL-NPs for 1, 3, 7 days and evaluated via MTT assay. No significant effect on cell viability was observed for all days tested with nanoparticle concentrations $(30 \mu \mathrm{g} / \mathrm{mL})$ (Fig. 6). The results of the hemolysis assay showed that all samples had permissible hemolysis. However, the nanoparticles with a concentration of $200 \mu \mathrm{g} / \mathrm{mL}$ showed higher hemolysis values (>10\%) compared to the positive control ( $0.5 \mathrm{~mL}$ of deionized water) (Fig. 7).

\section{NP Uptake and Neural differentiation of TMMSCs}

The NPs uptake by TMMSCs cells was evaluated following 2 hours of incubation by fluorescent imaging. It could be observed that the fluorescence of the curcumin-loaded PCL- NPs (yellow) is located in the cytoplasm around the nucleus (blue, stained by DAPI), indicating that the PCL-NPs is internalized into the cells (Fig. 8).

According to $\mathrm{qPCR}$ analysis, nestin expression was significantly lower in RA-loaded NPs than RA (10uM) and RA $(0.2 \mu \mathrm{M})$. On the other hand, the B-tubulin gene expression was markedly higher in RA-loaded NPs $(1.411 \pm 0.021$-fold, $\mathrm{p} \leq 0.0001)$ and (2.591 fold, $\mathrm{p} \leq 0.0001)$ compare to the NPs and RA $(0.2 \mu \mathrm{M})$, respectively.

MAP-II gene expression was significantly higher in RA-loaded NPs (3.819 fold, $\mathrm{p} \leq 0.0001)$ and $(2.757$ fold, $\mathrm{p} \leq 0.0001)$ compared to RA $(0.2 \mu \mathrm{M})$ and NPs, respectively (Fig. 9).

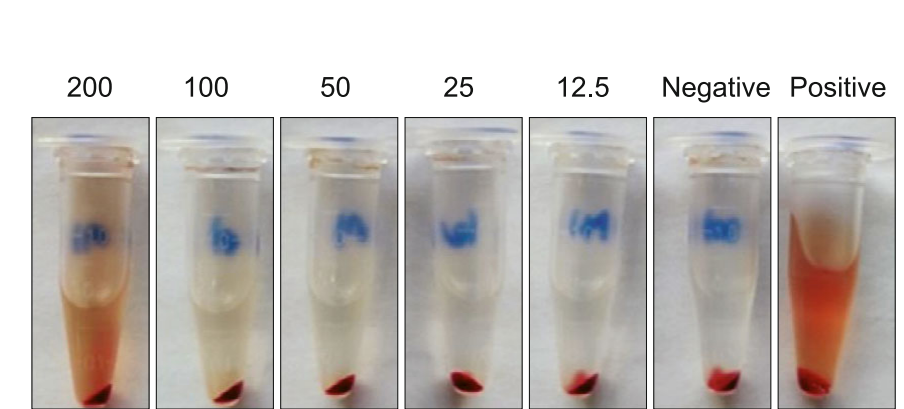

A

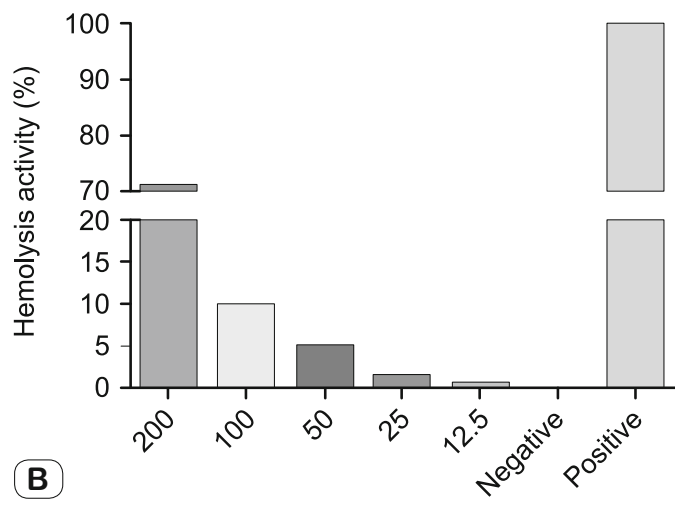

PCL-NPs concentration

Fig. 7. Hemolysis assay. A: visual inspection of the tubes containing diluted total blood after exposure to PCL-NPs. B: Percentage of hemolysis induced by PCL-NPs (Concentrations between $200 \mu \mathrm{g} / \mathrm{ml}$ and $12.5 \mu \mathrm{g} / \mathrm{ml}$ ).
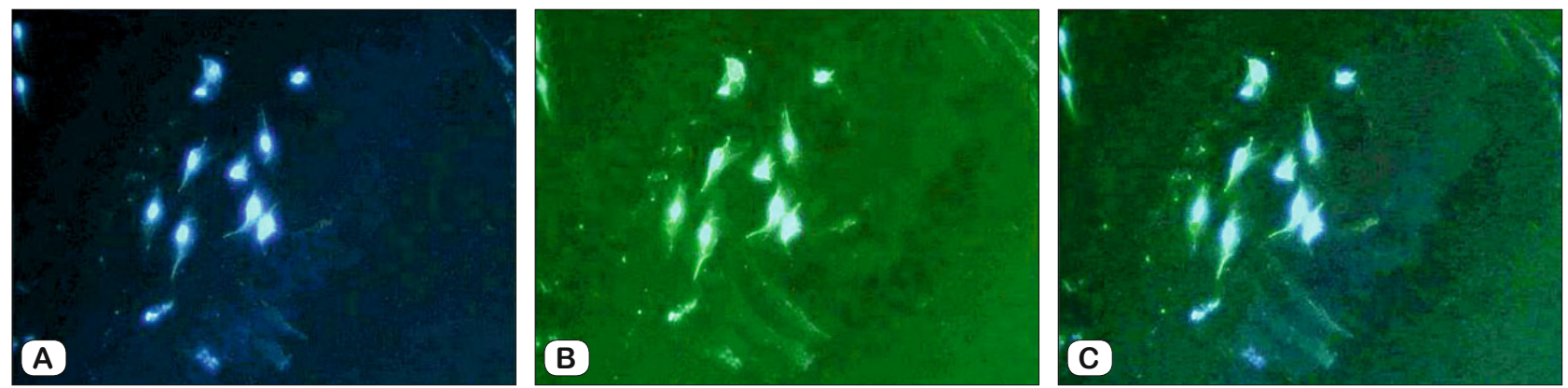

Fig. 8. Cell uptake analysis. The cells were stained by DAPI (blue) and the curcumin-loaded nanoparticles (yellow). A: left image from DAPI channel; B: center image from the yellow fluorescent channel; C: right image from combined green fluorescent protein channel and DAPI channel. 
The immunofluorescence analysis showed the expression of neural markers, including $\beta$ tubulin III and MAP-2 at the protein level in both RA $(10 \mu \mathrm{M})$ and RA-loaded NPs after 7 days of differentiation (Fig. 10).

\section{Discussion}

The polymeric nanoparticle (PNP) has shown to be a suitable option for growth factor delivery. Furthermore, the microfluidic systems revealed the ability to overcome the disadvantages and limitations represented by the conventional methods of NPs synthesis $(6,11,32)$. Therefore, in the present study, RA-loaded NPs were synthesized using a microfluidic system to enhance the entry of RA and induction of TMMSCs differentiation to neural-like cells.

To promote the quality of NPs by microfluidic systems, the design parameter of the microchip, such as channels diameter, length, and angles, the local position must be adopted to the desired

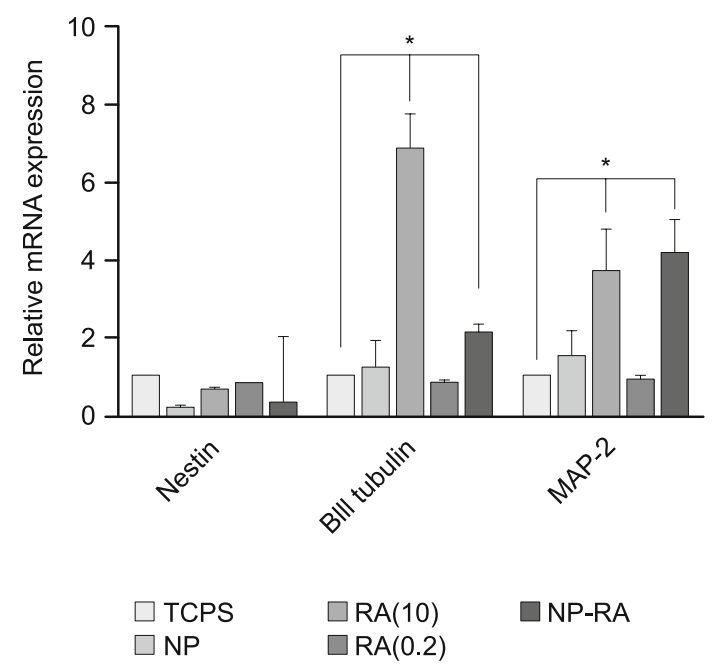

Fig. 9. qPCR analysis. The column ratio is the expression rate of genes compared to cultivated TMMSCs on TCPS. The GAPDH was used as an endogenous control for relative quantification. Data are represented as mean \pm SD. Asterisks show significance $\mathbf{p} \leq \mathbf{0 . 0 0 1}$. condition, including pressure, temperature, chemical compatibility, and concentration (33). Here, the results of synthesized NPs have approved that the microchip has been designed and fabricated in a suitable adoption to the employed operational conditions. On the other hand, microfluidic systems can decrease the exposure of induction factors to the rough situation during processing, an essential issue in delivery systems (7,11-13).

In the present study, the PCL NPs were synthesized using microchips, and the physical-chemical properties were characterized. The results showed that the microfluidic system could precisely control the size, PDI, morphology, and increased reproducibility of NPs synthesis.

The size and surface charge are the most important physicochemical properties of NPs that determined cell interactions and cellular uptake (34). It was declared that the therapeutic effects of RA-loaded PCL NPs would depend on internalization and drug release of the NPs by target cells. Here, the cellular uptake assay showed the internalization of RA-loaded NPs into the TMMSCs. The researchers reported that the maximum uptake rate of NPs in human retinal pigment epithelium (ARPE-19) cells were increased as particles' size decreased from $250 \mathrm{~nm}$ to $50 \mathrm{~nm}$ (31). However, in agreement with our study, the result showed that microfluidic systems had synthesized the suitable size of NPs $(120 \mathrm{~nm})$ for cellular uptake. However, in this study, the charge of PCL-NPs synthesis is negative and penetrates TMMSCs two hours after incubation. Several studies have established that NPs with negative charges can competently overwhelm the anionic cell membrane and uptake to cells (35-37). This phenomenon could be attributed to the fact that NPs surface charges are dynamic and may change over time in reaction to biological and environmental situations that resulted in some unintentional biological responses of NPs and therapeutic outcomes (34).

PCL is one of the most common Food and Drug Administration (FDA) approved biopolymers used in delivery systems. In the present study, the MTT assay results showed that PCLNPs $(30 \mu \mathrm{g} / \mathrm{mL})$ had no significant effect on cell viability. In agreement with our research Lin et al (38) showed that PCL-NPs $(25 \mathrm{ug} / \mathrm{ml})$ had no significant cytotoxicity on cells for up to 4 days. Besides, contrary to our study on day 6 , they reported that
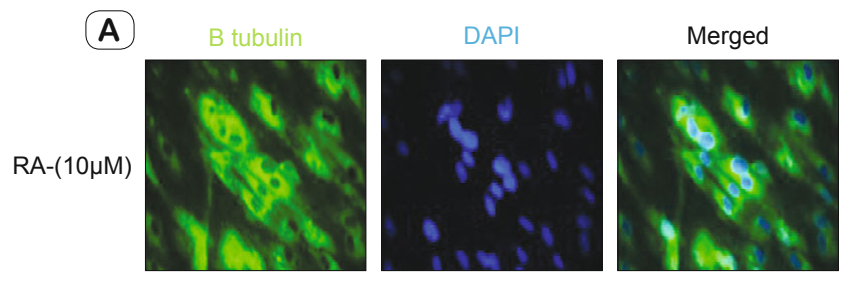

(B)
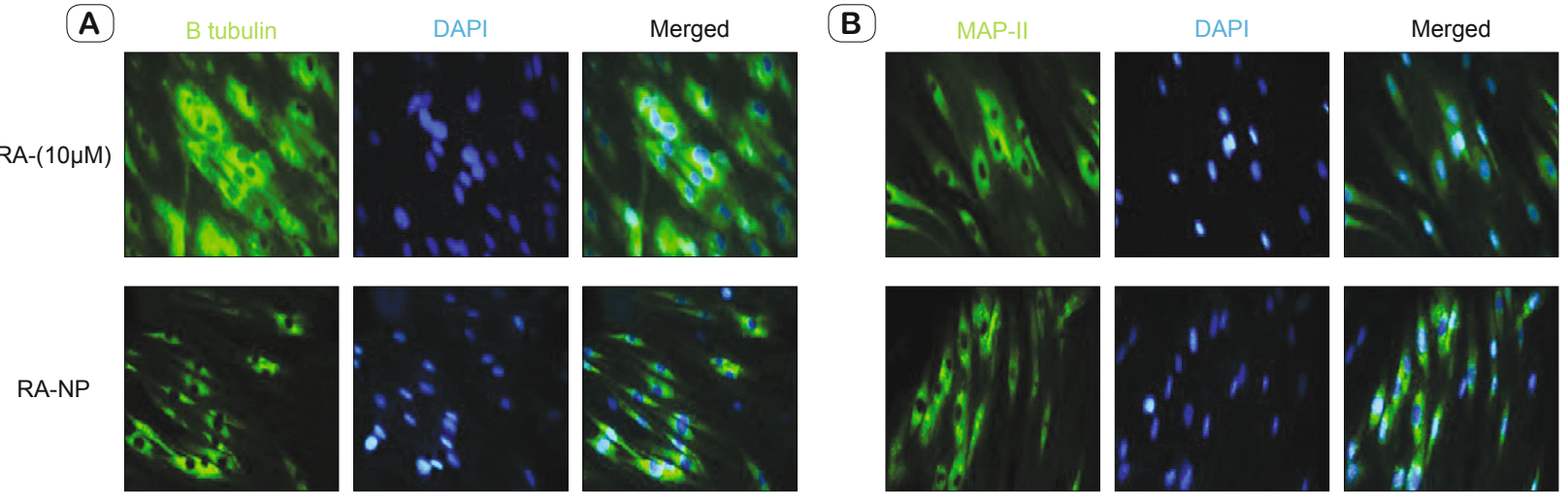

Fig. 10. Immunostaining analysis. The TMMSCs were cultivated and exposed with RA, PCL-NPs for 7 Day and the expression of $\beta$ tubulin III (A) and MAP-II (B) proteins was analyzed. 
PCL-NPs reduced viability in retinal pigment epithelium cell line (ARPE-19). This difference could be attributed to the diversity between the cell type, NPs concentration, and NPs fabrication method. Another critical element in the development of NPs systems is blood compatibility. The interaction of materials with blood components can cause lysis of erythrocytes. The hemolysis assay results showed that the PCL-NPs concentrations lower than $50 \mu \mathrm{g} / \mathrm{ml}$ decreased the blood lysis.

In the present study, the RA was loaded in PCL-NPs at $7.8 \%$ using the microfluidic method. The FTIR analysis revealed the presence of RA in the fabricated PCL-NPs without changing chemical composition. The result confirms the efficiency of NPs in the drug's local delivery. Only $25 \%$ of loaded RA was released in the first 4 hours, and more than $70 \%$ of the loaded RA remained in NPs formulation because the fabricated NPs could internalize in the cell after $2 \mathrm{~h}$. The other same study showed that high stability and controlled release of nanoparticles during cell uptake cause internalization of more drugs into the cell (39). It was stated that the RA release profile from NPs has a content-dependent manner due to its hydrophobic nature (40). Inconsistent with our study, Shakiba and co-workers reported more than $80 \%$ of loaded RA $(10 \% \mathrm{w} / \mathrm{v})$ released in the first 3 days from a PCLPEG-PCL micelle (40).

In the present study, the RA molecule delivered by PCLNPs induced the neuronal differentiation of TMMSCs. The result showed that the expression of neuronal marker genes, including Beta-tubulin III and MAP-2, increased in RA-loaded PCL NPs. Beta- tubulin III and MAP-2 are known as the neuron-specific markers that promote the microtubule network's assembly and stability, respectively $(41,42)$.

Beta- tubulin III and MAP-2 are immature postmitotic neurons and mature neural markers, respectively, used as in vitro and in vivo neural detection $(43,44)$. Although most in vitro studies demonstrated that RA could promote neurogenesis in stem cells (45), high concentration of RA $(>10 \mu \mathrm{M})$ has a toxicological effect on cells (46). Due to this limitation, finding new methods for transferring low RA concentrations into stem cells could positively impact cell viability and neural differentiation. In this study, NPs loaded with a low concentration of RA using microfluidic systems and their effects on the neural differentiation of TMMSCs were examined. However, the result showed high expression of neural markers ( $\beta$ - tubulin III and MAP-2) documented in TMMSCs after treating RA-loaded PCL NPs $(0.2 \mu \mathrm{M}$ loading RA). On the other hand, the results showed that fabricated NPs using microfluidic systems could internalize RA into stem cells with a low amount of RA concentration, transfer to the cytoplasm - nuclear, and differentiated stem cells toward neuron-like cells.

\section{Study highlights}

Fabricated retinoic acid-loaded PCL Nanoparticles using microfluidic systems could internalize a low amount of Retinoic acid concentration into stem cells for differentiation into neuronlike cells.

\section{References}

1. Jin Q, Deng $\mathbf{Y}$, Chen $\mathbf{X}$ et al. Rational design of cancer nanomedicine for simultaneous stealth surface and enhanced cellular uptake. Acs Nano 2019; 13: 954-977.

2. Liang J, Yan H, Puligundla P et al. Applications of chitosan nanoparticles to enhance absorption and bioavailability of tea polyphenols: A review. Food Hydrocolloids 2017; 69: 286-292.

3. Cupic KI, Rennick JJ, Johnston AP et al. Controlling endosomal escape using nanoparticle composition: current progress and future perspectives. Nanomedicine 2019; 14: 215-223.

4. Bai X, Wang S, Yan $X$ et al. Regulation of cell uptake and cytotoxicity by nanoparticle core under the controlled shape, size, and surface chemistries. ACS nano 2019; 14: 289-302.

5. Ban C, Jo M, Park YH et al. Enhancing the oral bioavailability of curcumin using solid lipid nanoparticles. Food Chem 2020; 302: 125328.

6. Rane AV, Kanny K, Abitha V et al. Methods for synthesis of nanoparticles and fabrication of nanocomposites. Synthesis of inorganic nanomaterials: Elsevier, 2018: 121-139.

7. Liu D, Cito S, Zhang Y et al. A versatile and robust microfluidic platform toward high throughput synthesis of homogeneous nanoparticles with tunable properties. Adv Materials 2015; 27: 2298-2304.

8. Shrimal P, Jadeja G, Patel S. Microfluidics nanoprecipitation of telmisartan nanoparticles: effect of process and formulation parameters. Chem Papers 2021; 75: 205-214.

9. Gangadoo S, Stanley D, Hughes RJ et al. The synthesis and characterisation of highly stable and reproducible selenium nanoparticles. Inorganic Nano-Metal Chem 2017; 47: 1568-1576.

10. Streck S, Neumann H, Nielsen HM et al. Comparison of bulk and microfluidics methods for the formulation of poly-lactic-co-glycolic acid (PLGA) nanoparticles modified with cell-penetrating peptides of different architectures. Internat J Pharm X 2019; 1: 100030.

11. Xu S, Nie Z, Seo M et al. Generation of monodisperse particles by using microfluidics: control over size, shape, and composition. Angewandte Chem 2005; 117: 734-738.

12. Edel JB, Fortt R, DeMello JC et al. Microfluidic routes to the controlled production of nanoparticles. Chem Comm 2002: 1136-1137.

13. Nakamura H, Tashiro A, Yamaguchi Y et al. Application of a microfluidic reaction system for CdSe nanocrystal preparation: their growth kinetics and photoluminescence analysis. Lab Chip 2004; 4: 237-240.

14. Karnik R, Gu F, Basto $P$ et al. Microfluidic platform for controlled synthesis of polymeric nanoparticles. Nano Lett 2008; 8: 2906-2912.

15. Whitesides GM. The origins and the future of microfluidics. Nature 2006; 442: 368-373.

16. Duncanson WJ, Lin T, Abate AR et al. Microfluidic synthesis of advanced microparticles for encapsulation and controlled release. Lab Chip 2012; 12: 2135-2145.

17. Xu J, Zhang S, Machado A et al. Controllable microfluidic production of drug-loaded PLGA nanoparticles using partially water-miscible mixed solvent microdroplets as a precursor. Sci Reports 2017; 7: 1-12.

18. Tang $\mathbf{Z}$, He $\mathbf{C}$, Tian $\mathbf{H}$ et al. Polymeric nanostructured materials for biomedical applications. Progr Polymer Sci 2016; 60: 86-128.

19. Lale SV, RG A, Aravind A et al. AS1411 aptamer and folic acid functionalized $\mathrm{pH}$-responsive ATRP fabricated pPEGMA-PCL-pPEGMA 
polymeric nanoparticles for targeted drug delivery in cancer therapy. Biomacromolecules 2014; 15: 1737-1752.

20. Cheng R, Meng F, Deng $C$ et al. Dual and multi-stimuli responsive polymeric nanoparticles for programmed site-specific drug delivery. Biomaterials 2013; 34: 3647-3657.

21. Lee K, Silva EA, Mooney DJ. Growth factor delivery-based tissue engineering: general approaches and a review of recent developments. J Royal Soc Interface 2011; 8: 153-170.

22. Bodakuntla S, Jijumon A, Villablanca $C$ et al. Microtubule-associated proteins: structuring the cytoskeleton. Trends Cell Biol 2019; 29: 804-819.

23. Nath SC, Horie M, Nagamori $\mathbf{E}$ et al. Size- and time-dependent growth properties of human induced pluripotent stem cells in the culture of single aggregate. J Biosci Bioengineering 2017; 124: 469-475.

24. Wu H, Zhao J, Fu B et al. Retinoic acid-induced upregulation of miR-219 promotes the differentiation of embryonic stem cells into neural cells. Cell Death Dis 2017; 8: e2953-e.

25. Lee OK, Kuo TK, Chen WM et al. Isolation of multipotent mesenchymal stem cells from umbilical cord blood. Blood 2004; 103: 1669-1675.

26. Lindvall O, Kokaia Z, Martinez-Serrano A. Stem cell therapy for human neurodegenerative disorders-how to make it work. Nat Med 2004; 10 Suppl: S42-50.

27. Luo Y, Liang C, Liu $\mathbf{Y}$ et al. RXR $\alpha$ and MRTF-A have a synergistic effect in the retinoic acid-induced neural-like differentiation of adult bone marrow-derived mesenchymal stem cells. Cell Biol Internat 2020; 44: 1373-1381.

28. Szuts EZ, Harosi FI. Solubility of retinoids in water. Arch Biochem Biophys 1991; 287: 297-304.

29. Nadri S, Yazdani S, Arefian E et al. Mesenchymal stem cells from trabecular meshwork become photoreceptor-like cells on amniotic membrane. Neurosci Lett 2013; 541: 43-48.

30. Zhong X, Ji C, Chan AK et al. Fabrication of chitosan/poly ( $\varepsilon$-caprolactone) composite hydrogels for tissue engineering applications. J Materials Sci: Materials Med 2011; 22: 279-288.

31. Varshosaz J, Hassanzadeh F, Sadeghi $\mathbf{H}$ et al. Retinoic acid decorated albumin-chitosan nanoparticles for targeted delivery of doxorubicin hydrochloride in hepatocellular carcinoma. J Nanomaterials 2013; 2013.

32. Jin S, Ye K. Nanoparticle-mediated drug delivery and gene therapy. Biotechnol Progress 2007; 23: 32-41.
33. Marre S, Jensen KF. Synthesis of micro and nanostructures in microfluidic systems. Chem Soc Rev 2010; 39: 1183-1202.

34. Donahue ND, Acar H, Wilhelm S. Concepts of nanoparticle cellular uptake, intracellular trafficking, and kinetics in nanomedicine. Adv Drug Deliv Rev 2019; 143: 68-96.

35. Ayala V, Herrera AP, Latorre-Esteves M et al. Effect of surface charge on the colloidal stability and in vitro uptake of carboxymethyl dextran-coated iron oxide nanoparticles. J Nnanoparticle Res 2013; 15: 1874.

36. Lee JS, Ankone M, Pieters $\mathbf{E}$ et al. Circulation kinetics and biodistribution of dual-labeled polymersomes with modulated surface charge in tumor-bearing mice: comparison with stealth liposomes. J Control Release 2011; 155: 282-288.

37. Zhou Y, Shi L, Li Q et al. Imaging and inhibition of multi-drug resistance in cancer cells via specific association with negatively charged CdTe quantum dots. Biomaterials 2010; 31: 4958-4963.

38. Lin H, Yue Y, Maidana DE et al. Drug delivery nanoparticles: Toxicity comparison in retinal pigment epithelium and retinal vascular endothelial cells. Semin Ophthalmol: Taylor \& Francis, 2016: 1-9.

39. Lázaro IA, Haddad S, Sacca $S$ et al. Selective surface PEGylation of UiO-66 nanoparticles for enhanced stability, cell uptake, and $\mathrm{pH}$-responsive drug delivery. Chem 2017; 2: 561-578.

40. Shakiba E, Khazaei S, Hajialyani $M$ et al. Preparation and in vitro characterization of retinoic acid-loaded poly( $\varepsilon$-caprolactone)-poly(ethylene glycol)-poly(E-caprolactone) micelles. Res Pharm Sci 2017; 12: 465-478.

41. Caccamo D, Herman M, Frankfurter A et al. An immunohistochemical study of neuropeptides and neuronal cytoskeletal proteins in the neuroepithelial component of a spontaneous murine ovarian teratoma. Primitive neuroepithelium displays immunoreactivity for neuropeptides and neuron-associated beta-tubulin isotype. Amer J Pathol 1989; 135: 801.

42. Dehmelt L, Halpain S. The MAP2/Tau family of microtubule-associated proteins. Genome Biol 2005; 6: 1-10.

43. Tanapat P. Neuronal cell markers. Mater. Methods 2013; 3: 196.

44. und Halbach OvB. Immunohistological markers for staging neurogenesis in adult hippocampus. Cell Tissue Res 2007; 329: 409-420.

45. Wang T-W, Zhang H, Parent JM. Retinoic acid regulates postnatal neurogenesis in the murine subventricular zone-olfactory bulb pathway. Development 2005; 132: 2721-2732.

46. Xu J, Wang H, Liang $\mathrm{T}$ et al. Retinoic acid promotes neural conversion of mouse embryonic stem cells in adherent monoculture. Mol Biol Reports 2012; 39: 789-795. 